\title{
Vaginal Natural Orifice Transluminal Endoscopic Surgery (vNOTES) for Low-grade Endometrial Cancer: The First Fifty Patients
}

\author{
alexander burnett ${ }^{1}$ and Martha Rojo ${ }^{1}$ \\ ${ }^{1}$ University of Arkansas for Medical Sciences
}

March 17, 2021

\begin{abstract}
Study Objective: To review our experience with low-grade endometrial cancer managed with vaginal natural orifice transluminal endoscopic surgery (vNOTES) to determine the feasibility of this approach. Design: Retrospective review of the first fifty cases of endometrial cancer treated by vNOTES Setting: Academic University Population: Fifty consecutive cases of vNOTES management of women with low-grade endometrial cancer were reviewed to detail patient characteristics, operative procedures and outcomes, complications and follow-up. Methods: Patients underwent vNOTES hysterectomy and bilateral salpingooophorectomy. If Mayo criteria for lymphadenectomy were met, a laparoscopic lymph node removal was performed. Main Results: Mean age was 63 (33-90); mean BMI was 35.4 (22-58). In 44 women, both tubes and ovaries were removed via vNOTES. Three women had their ovaries retained due to early age, one woman had exploration for removal of an ovary with metastatic disease, and two women had neither tubes nor ovaries removed due to atresia and inability to identify these organs. Two patients underwent laparoscopic lymphadenectomy on the bases of frozen section evaluation. Mean operative time was 98 minutes (30-215). Fourteen patients were discharged the day of surgery; 34 discharged after overnight observation; hospitalization was prolonged in one patient due to ileus and one patient underwent laparotomy 30 hours after vNOTES for bleeding. Conclusion: VNOTES is a feasible management strategy for women with low-grade endometrial cancer. It permits laparoscopic inspection of the abdomen and direct visualization of vascular pedicles. It can provide a rapid recovery with minimal discomfort and no visible scar.
\end{abstract}

\section{Introduction}

Endometrial cancer is the most common malignancy in Gynecologic Oncology. The mainstay of treatment per National Comprehensive Cancer Network (NCCN) guidelines is for removal of the uterus, fallopian tubes and ovaries [1]. Lymph node assessment may be performed either by Sentinel lymph node removal or based on the pathologic findings of the cancer in the uterus. Guidelines also recommend utilizing a minimally invasive approach when feasible.

The American College of Obstetricians and Gynecologists and the American Association of Gynecologic Laparoscopists recommend the vaginal route for hysterectomy as this provides the least morbidity, quickest recovery and no visible scars for the patient $[2,3]$. Despite these recommendations, vaginal hysterectomy is rarely used in endometrial cancer for a variety of reasons. First, visual inspection of the abdomen is not possible; second, the ovaries can be difficult to visualize and remove particularly in the postmenopausal woman; third, lymph nodes cannot be assessed; and fourth, surgical expertise in transvaginal hysterectomy has diminished with the increased use of laparoscopic procedures.

Vaginal Natural Orifice Transluminal Endoscopic Surgery (vNOTES) is a newer surgical approach that employs laparoscopic techniques through the vagina [4]. This method permits abdominal exploration, resection 
of the fallopian tubes and ovaries under direct visualization at the infundibulo-pelvic ligament, and pelvic washings can be obtained. The procedure can be performed as outpatient surgery, requires minimal or no narcotics postoperatively, has minimal wound complications and no visible scar. A systematic review and a randomized trial have shown this technique to be non-inferior to standard laparoscopy for hysterectomy [5, $6]$.

We began performing vNOTES for endometrial cancer in July 2017. This procedure is offered for low-grade endometrial cancer provided the following criteria are met: 1) the uterus is less than 12 weeks size, 2) there is no obvious metastatic disease, and 3) the patient is willing to undergo laparoscopic lymph node assessment if high-risk features are present on frozen section of the uterus. We use the Mayo criteria for deciding whether or not to perform lymph node removal [7]. The purpose of this report is to review the first 50 consecutive patients who underwent vNOTES for a pre-operative diagnosis of grade 1 or 2 endometrioid adenocarcinoma of the uterus.

\section{Materials and methods}

\section{Patients}

Fifty consecutive women with a diagnosis of low-grade endometrial cancer constitute this report. Age, Body Mass Index (BMI), parity and prior surgery were not exclusionary criteria. Patients were counseled that if surgical difficulties were encountered they may require either laparotomy or laparoscopy to complete the procedure. Also, if high-risk features were found that warranted lymph node removal, this would be performed with abdominal laparoscopy. This review was approved by the University Institutional Review Board. Preoperative and postoperative pathology was reviewed by a gynecologic pathologist.

\section{Technique}

The description of the procedure for hysterectomy and bilateral salpingo-oophorectomy has been extensively reviewed elsewhere $[5,6]$.

\section{Post-operative Management}

After the procedure patients were given the option of being discharged home that day or staying in the hospital overnight for observation. They were encouraged to utilize nonsteroidal anti-inflammatory agents for pain control and were discharged home with no more than eight tablets of a low-dose opioid. They were permitted to drive once they were no longer using opiates and vaginal rest continued for approximately five weeks postoperatively. They returned to the clinic in two weeks to discuss the final pathology.

\section{Results}

Fifty consecutive cases of low-grade endometrial cancer underwent vNOTES between July 2017 and November 2019. Patient characteristics are listed in table 1. The mean patient age was 63 years (33-90); the mean BMI was 35.4 (22-58). Twelve of the patients had had no prior abdominal surgery. Of the remaining 38,10 had had prior cesarean deliveries including five with two or more prior cesarean deliveries. The remaining 28 had a myriad of other abdominal or pelvic surgeries, both major and minor. The most frequent comorbidities were hypertension and non-insulin-dependent diabetes. Only four of the patients had no major comorbidities. Preoperative pathology revealed grade 1 endometrioid adenocarcinoma in 46 women and grade 2 endometrioid adenocarcinoma in four women.

Operative procedures (Table 2) included the following: 44 women underwent vNOTES hysterectomy and bilateral salpingo-oophorectomy, three women underwent vNOTES hysterectomy with bilateral salpingectomy and ovarian preservation due to young age, one woman underwent vNOTES hysterectomy with left salpingo-oophorectomy followed by laparotomy for right salpingo-oophorectomy due to the appearance of the right tube and ovary, and two women underwent vNOTES hysterectomy alone as the bilateral tubes and ovaries were atretic and could not be visualized. Additional surgery performed included laparoscopic lymphadenectomy in two patients, vNOTES upper abdominal lysis of adhesions in two patients, and one patient undergoing vNOTES appendectomy. 
The mean operative time as measured by incision in the cervix to closure the vaginal cuff or completion of any abdominal surgery was 98 minutes (30-215). Estimated blood loss was reported as less than $100 \mathrm{~mL}$ in 47 patients, 200 to $299 \mathrm{~mL}$ in two patients, and $300 \mathrm{~mL}$ in one patient. The mean uterine weight was 111 gm $(27-436)$.

Fourteen women were discharged home the day of surgery, 34 were discharged home the following day after observation. Postoperative ileus led to a three night stay for one patient, and one patient underwent a laparotomy for bleeding 30 hours after her vNOTES surgery and was in the hospital a total of three nights.

\section{Additional Procedures}

Two patient underwent abdominal laparoscopic lymphadenectomy at the time of surgery due to high-risk features on frozen section. One was diagnosed with three of 14 pelvic lymph nodes positive, the other had negative pelvic lymph nodes. One patient had a vNOTES appendectomy due to scarring of the appendix around the right adnexae. In one patient we elected to perform a laparotomy due to purulent material coming from the right tube and ovary. This was found to be metastatic disease from a deeply invasive endometrial cancer.

No patient required conversion to abdominal approach for hysterectomy.

\section{Complications}

There were three complications associated with these 50 cases. One woman was admitted to the emergency department five days after her surgery with abdominal pain. She underwent laparotomy and was found to have a thermal injury of her small intestine which was over sewn and subsequently recovered uneventfully. This patient was a nulligravida with a BMI of 48 and was chronologically the fifth vNOTES performed.

The second complication occurred in a slender woman who underwent resection of the uterus, fallopian tubes and ovaries without difficulty and video documented excellent hemostasis at the completion of the procedure. About 15 hours after her surgery she had what she described as "a violent episode of vomiting" and then began to develop right upper quadrant pain. Her hemoglobin began to fall over the course of the day and she was subsequently taken for an exploratory laparotomy where there was active bleeding from her right ovarian vessels. These were ligated and she was discharged home the following day.

The third complication occurred when a patient developed vaginal bleeding in the recovery room. She was taken back to the operating room and had a bleeding site on the vaginal cuff ligated. She was discharged home the following day.

\section{Pathology}

Final pathology is listed in table 3. 37 patients had a final pathology of grade 1 endometrioid adenocarcinoma; 32 of these were stage IA, four were stage IB, and one was stage IIICI. 10 patients were found to have a grade 2 endometrioid adenocarcinoma, seven of whom were stage IA, one was stage II, and two were stage IIIA. Two patients were found on final pathology to have a grade 3 endometrioid adenocarcinoma, neither of whom had invasion into the myometrium. Finally, one patient on final pathology was found to have a dedifferentiated carcinoma extending into the cervical stroma consistent with stage II. Additional Therapy

One of the patients with noninvasive grade 3 cancer recurred five months after surgery and was treated with chemotherapy. She subsequently died of metastatic disease 18 months after her initial surgery. Three patients were treated with adjuvant pelvic radiation. The patient with dedifferentiated carcinoma received chemotherapy plus radiation. One patient with bilateral ovarian involvement received adjuvant chemotherapy alone. Forty-nine of the 50 patients are currently without evidence of disease with a median follow-up of 29 months.

\section{Discussion}

Vaginal hysterectomy has the lowest morbidity when compared to laparoscopic or open procedures $[8,9,10]$, but Gynecologic Oncologists have rarely adapted this approach for endometrial cancer. The typical patient 
with low-grade endometrial cancer, or type I disease, is obese, elderly and has multiple medical comorbidities. Our present study has confirmed the feasibility of performing vNOTES in women with low-grade endometrial cancer and may represent a particularly attractive method for these patients.

The major arguments against a transvaginal approach for endometrial cancer include difficulties in removing the adnexa, inability to evaluate the upper abdomen, cannot perform a pelvic washing or lymphadenectomy. The vNOTES procedure is able to overcome these limitations. The adnexa can be safely removed with direct laparoscopic visualization of the ovarian vasculature. Finally, both Sentinel lymph nodes and pelvic lymphadenectomy can be performed via vNOTES but were incorporated into the author's skill set after the first fifty cases $[11,12]$.

In a previous study retrospectively comparing vaginal hysterectomy with robotic hysterectomy for endometrial cancer [13], the vaginal group had a lower operative time, better overall survival at five years, and lower cost than the robotic group. Lymph node removal was performed in the vaginal group for high-risk features (deep invasion, higher grade, large tumor) by either laparoscopy or robotic approach. The authors reflect that upper abdominal exploration and pelvic washings cannot be performed with the vaginal hysterectomy. They do not present data on how successful removal of the tubes and ovaries was from this approach.

There were three complications which occurred in this cohort. The first in a nulliparous woman with a BMI of 48 who suffered a thermal injury to the small bowel and required laparotomy with over-sew of the injury five days postoperatively. She was the fifth patient to undergo this procedure and probably represented a greater surgical challenge than our experience at that time warranted. The second complication involved an immediate postoperative bleeding from the vaginal cuff which was remedied quickly. The third complication was delayed bleeding from the ovarian vessels likely as a result of increased abdominal pressure during vomiting approximately 12 hours postoperatively.

Postoperative recovery was similar to a standard vaginal hysterectomy with $28 \%$ of patients going home on the day of surgery and all but two of the remaining discharged home the following morning. Narcotic use was minimal. At the two week postoperative visit all patients were off any pain medication.

This is the first reported series of utilizing vNOTES for low-grade endometrial cancer. The strength of this study is that it reflects the initial experience with the first 50 consecutive cases. It reveals the feasibility of vNOTES regardless of patient BMI, prior pregnancy status, prior surgical status or uterine descent. Recovery appears similar to transvaginal hysterectomy and requires minimal narcotics and no visible surgical scar. While cost was not specifically evaluated, the equipment and operating room time were similar to a single incision laparoscopy.

The limitations of this study are that it represents a single surgeon's experience and therefore may not be applicable to all practitioners. The other major limitation of this technique was the author's inability to evaluate lymph nodes from this approach with the first fifty patients. However, as several authors worldwide have documented both Sentinel node resection and pelvic and even para-aortic lymphadenectomy via vNOTES, these techniques will be incorporated with increased experience.

In summary, vNOTES approach is feasible for patients with low-grade endometrial carcinoma. It may combine the safety and comfort of the transvaginal approach with the visualization, magnification and instrumentation of the laparoscopic approach. Because the majority of the surgery is performed using laparoscopic techniques, this approach could be adapted by any surgeon who currently performs minimally invasive procedures.

\section{Disclosure of Interest}

Neither author as financial, personal, political, intellectual, or religious interests related to this publication.

\section{Contribution of authorship .}

Dr. Burnett performed all the procedures detailed in this study. He was responsible for the conception, planning and carrying out the patient care. He also contributed to the analyzing and writing up of the work. 
Dr. Rojo contributed to the analyzing and writing up of the work.

\section{Ethics approval.}

This retrospective analysis was approved by the UAMS IRB on April 29, 2020. The approval letter is attached after the references.

\section{Funding.}

No funding was allocated for the performance of this study.

\section{Table of legends:}

Table 1. Pre-operative characteristics $(\mathrm{n}=50)$

Table 2. Operative Procedures

Table 3. Final Pathology

\section{References}

1. NCCN Clinical Practical Guidelines in Oncology. Uterine Neoplasms. Version 1.2020 - March 06, 2020. https://www.nccn.org/professionals/physician_gls/pdf/uterine.pdf

2. ACOG Committee Opinion 701. Choosing the route of hysterectomy for benign disease. Committee Opinion 701 . June 2017

3. AAGL position statement: route of hysterectomy to treat benign uterine disease. JMIG 2011; 18: 1-2

4. Baekelandt J. Total vaginal NOTES hysterectomy: a new approach to hysterectomy. JMIG 2015; 22 : 1088-1094

5. Li C, Hua K. Transvaginal natural orifice transluminal endoscopic surgery (vNOTES) in gynecologic surgeries: a systematic review.Asian J Surgery 2020; 43, 44-51

6. Baekelandt JF, De Mulder PA, Le Roy I, Mathieu C, Laenen A, Enzlin P, Weyers S, Mol BWJ, Bosteels JJA. Hysterectomy by transvaginal natural orifice transluminal endoscopic surgery versus laparoscopy as a day-care procedure: a randomized controlled trial. BJOG 2019; 26, 105-113

7. Convery PA, Cantrell LA, Di Santo N, Broadwater G, Modesitt SC, Avarez Secord A, Havrilesky LJ. Retrospective review of an intraoperative algorithm to predict lymph node metastasis in low-grade endometrial adenocarcinoma. Gyn Oncology 2011; 123, 65-70

8. Aarts JWM. Nieboer TE, Johnson N, Tavender E. Garry R, Mol BWJ,. Klulvers KB. Surgical approach to hysterectomy for benign gynecological disease (Cochrane Review). Cochrane Database of Systematic Reviews 2015; 8

9. Schmitt JJ, Leon DAC, Occino JA, Weaver AL, Dowdy SC, Bakkum-Gamez JN, Pasupathy KS, Gebhart JB. Determining optimal route of hysterectomy for benign indications. Obstet Gynecol 2007;1289, $130-8$

10. Sandberg EM, Twijnstra ARH, Driessen SRC, Jansen FW. Total laparoscopic hysterectomy versus vaginal hysterectomy: a systematic review and meta-analysis. JMIG 2017;24, 206-217

11. Tantitamit T, Lee CL. Application of sentinel lymph node technique to transvaginal natural orifices transluminal endoscopic surgery in endometrial cancer. JMIG 2019; 26, 949-953

12. Baekelandt JF. New retroperitoneal transvaginal natural orifice transluminal endoscopic surgery approach to sentinel node for endometrial cancer: a demonstration video. JMIG 2019; 26, 1231-1232

13. Nitschmann CC, Multinu F, Bakkum-Gamez JN, Langstraat CL, Occhino JA, Weaver AL, Cliby WA, Mariani A, Dowdy SC. Vaginal vs. robotic hysterectomy for patients with endometrial cancer: a comparison of outcomes and cost of care. Gyn Oncology 2017; 145, 555-561

Institutional Review Board4301 West Markham, \#636 Little Rock, AR 72205-7199 501-686-5667 501686-7265 (fax) http://irb.uams.edu/ FWA00001119 04/29/2020PI Name: Burnett, AlexanderPI Department: COM OB \& GYN Gynecologic OncologyProtocol Number: 261036Protocol Title: A Retrospective Review of vNOTES for Management of Low-grade Endometrial Cancer NEW SUBMISSION EXEMPT The Institutional Review Board classified this study as Exempt, category [4c], on 04/29/2020, 
based on Title 45 CFR 46.101. The IRB granted a waiver of HIPAA authorization for the PHI described in the submission as follows: Clinical and pathological information from women diagnosed with grade 1 or 2 endometrial cancer treated with vNOTES. This includes: 1. Patient characteristics including age, BMI, parity, prior surgery, and pre-operative tumor characteristics. 2. Surgical procedures including operative time, blood loss, complications and postoperative recovery as well as ultimate pathology. The IRB determined that the research cannot practicably be conducted without access to or use of this PHI, and cannot practicably be carried out without the waiver. The IRB determined that the research uses the following methods to ensure minimal risk to privacy of subjects:

- A plan to protect the identifiers from improper use or disclosure.

- A plan to destroy the identifiers at the earliest opportunity consistent with the conduct of research, unless there is a health or research justification for retaining the identifiers or retention is required by law.

- An assurance that the PHI will not be re-used or disclosed to any other person or entity, except as required by law, for authorized oversight of the research project, or for other research as permitted by the HIPAA regulations.

Reminder: All other HIPAA requirements, including Minimum Necessary Rule, still apply.

\section{Hosted file}

Table 1 Patient Characteristics.pdf available at https://authorea.com/users/402171/articles/ 514047-vaginal-natural-orifice-transluminal-endoscopic-surgery-vnotes-for-low-gradeendometrial-cancer-the-first-fifty-patients

\section{Hosted file}

Table 2 Operative Characteristics.pdf available at https://authorea.com/users/402171/ articles/514047-vaginal-natural-orifice-transluminal-endoscopic-surgery-vnotes-for-lowgrade-endometrial-cancer-the-first-fifty-patients

\section{Hosted file}

Table 3 Final Pathology.pdf available at https://authorea.com/users/402171/articles/ 514047-vaginal-natural-orifice-transluminal-endoscopic-surgery-vnotes-for-low-gradeendometrial-cancer-the-first-fifty-patients 